Scientific Visualization, 2020, volume 12, number 5, pages $61-78$, DOI: 10.26583/sv.12.5.06

\title{
Optical Fiber Splicing Defect Segmentation Using Hybrid Active Contour
}

\author{
A. Azizi ${ }^{1, A, B}, Z$. Azizi ${ }^{2, A}$ \\ A Department of Electrical Engineering, University of Mohamed Khider, Biskra, Algeria \\ в Laboratory of Identification, Command, Control and Communication 'LI3C' University \\ of Mohamed Khider, Biskra, Algeria \\ ${ }^{1}$ ORCID: 0ooo-0001-5151-8975, aziziabdallah87@yahoo.com \\ 2 ORCID: 0000-0002-4632-1825, azizizineb93@gmail.com
}

\begin{abstract}
In this paper, we are interested to segment splicing hot (infra-red) images using active contours in order to extract defects for the fiber splicing process on fiber splicer machine. The segmentation is assured by using a hybrid edge and region active contour; where region information uses local statistics to solve the problem of highlight or intensity inhomogeneity, whereas edge information based on Local Binary Patterns LBP, smoothes homogeneous regions and enhance contour information. Experimental results on hot images captured from the fiber splicer machine illustrate the effective performance of the proposed method.
\end{abstract}

Keywords: Active Contours, Defect segmentation, Edge indicator, Fiber Splicing, Local binary pattern.

\section{Introduction}

Recently, fiber optic transmission has become one of the most popular choices for longdistance fixed communication links due to its technology that provides a higher capacity data transfer at extremely high speeds. In many situations splicing often is required to connect the separated ends of fiber together to create a continuous optical path for transmission of optical signal from one fiber length to another. One of the basic fiber interconnection methods is fusion splicing by a Fiber Splicing Machine (FSM). As shown in Fig. 1, a fiber is made up of the core and the cladding those will be spliced, and the buffer that will be removed during the stripping, when splicing the fibers. However, to maintain high quality transmission, a good splice with low loss is required [1].

The splice loss is a power metric of the input and the output light across the fiber that can be estimated directly from the splicer machine. However, engineers usually prefer the use of the Optical Time Domain Reflectometer (OTDR) to measure the splice loss rather than using the evaluation result of the FSM. This is because the output of the splicer is only an approximate estimation [2]. For that, in this work, we propose to use image processing for the segmentation of splicing defects from the digital images captured from the splicer machine.

Many research works have been published in the field of defect segmentation. In the field of radiographic weld defects image segmentation, traditional techniques have been proposed such as thresholding and morphological approaches [3-5]. Recently, optimization techniques are introduced which tries to segment images by optimizing some criterion [6-8]. Active Contours [9-18] are the most popular techniques in this category where the idea is to drive an initial curve inside the image domain to be segmented to reach the boundaries of the objects of interest by minimizing energy where the curve is the argument of this energy [19]. Generally, active contours can be classified into edge-based models relying on contour information [9- 
11], region based models relying either on global or local image statistics [12-15] and hybrid models combining all information [16-18].

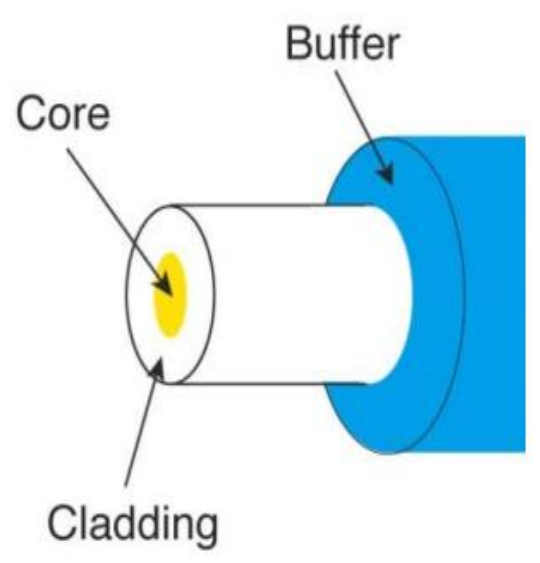

Fig. 1. Profile of the fiber.

Over the past few years, modern approaches based on convolutional neural networks (CNNs) [20-22] have yielded a new generation of image segmentation models with remarkable performance improvements. Image segmentation can be formulated as a classification problem of pixels with semantic labels (semantic segmentation) or partitioning of individual objects (instance segmentation). Semantic segmentation performs pixel-level labeling with a set of object categories (e.g., human, car, tree, sky) for all image pixels, thus it is generally a harder undertaking than image classification, which predicts a single label for the entire image [23]. In the field of fiber splicing defect segmentation, to our knowledge, there is no literature studied this problem except the work of Liu et al. [2,24] where the authors proposed to utilize both the Gaussian Mixture Model (GMM) and the Graph Cut Model (GCM) to solve the defect segmentation problem of the hot image on the splicer machine. The GMM is used to restrain the highlight of the defect images caused by LED lamp when collecting the image data during the splicing process by the machine camera. Then the GCM is employed to segment the defect region [24].

Although the GMM is a good solution for the highlight or intensity inhomogeneity phenomenon, however the standard GMM parameters are just estimated by some selected normal mode image whose image qualities are good; this makes not all the segmented region can be regarded as serious defects which will influence the final splicing effect [2]. In addition, Expectation Maximization (EM) algorithm used to estimate the GMM parameters is complex and unable in implementation for real-time applications [25].

In this work, in order to solve both the problem of highlight or intensity inhomogeneity and defect segmentation simultaneously, we combine the hybrid active contour proposed in [18] that uses local region information with the edge detection method based Local Binary Patterns (LBP) proposed in $[11,26]$ in a single model. Local region information is an effective way to deal with intensity inhomogeneity or highlight problem, whereas edge information based on LBP, smoothes homogeneous regions and enhance contour information.

The rest of this paper is organized as follow: in section 2, the fiber splicing process vision system is discussed with its diagram. Section 3 reviews related segmentation methods. Section 4 describes the proposed model. Experimental results on hot images are the objective of Section 5. Finally, Section 6 concludes the paper. 


\section{Fiber splicing machine vision system}

\subsection{Fiber splicing process}

The working diagram of the splicer machine is shown in Fig. 2. Its structure includes three parts: the fiber splicing system, the lamps and cameras system. When this splicing system works, first the fiber splicing component will heat the two fibers by current constantly. Second the two cameras fixed in the vertical and the horizontal directions of spliced fibers will capture two types of images; the visible image in the beginning and at the end of the splicing process, and the hot images during the entire splicing process. To improve the imaging definition of hot image, two lamps fixed in the opposite sides of cameras will cast rays into the fibers surfaces. Third, the two cameras will capture and send splicing images as live video displayed on the machine monitor (screen) [2].

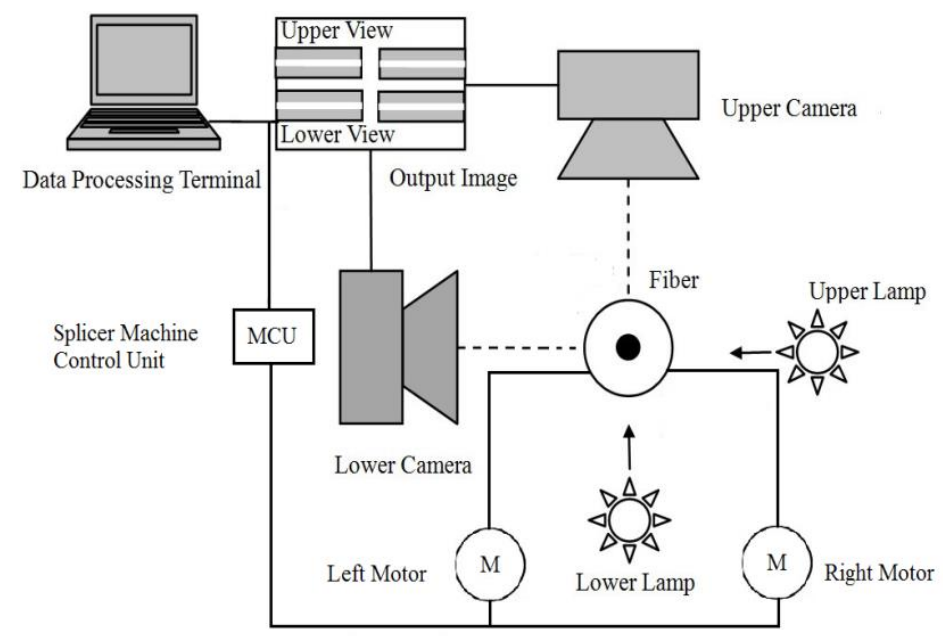

Fig. 2. The working diagram of the fiber splicing process.

\subsection{Fiber splicing machine image analysis}

As we discussed above, the visible images captured from the two cameras are used in two stages; the first one is in the beginning of splicing process before heating the fibers, where the visible images are treated by the fiber splicer machine control unit. This first processing aimed at aligning and pushing fibers to be closed to each other via the left and the right motors as shown in Fig. 2.

The second one is at the end of the splicing process where visible images treated by the machine control unit are used in order to detect typical splicing defects namely: bubbles, line, thin fiber, fat fiber, and separated fibers. In addition to other defects at the first stage (in the beginning) which are large cleave angle and fibers cores mismatch. Fig. 3 shows some samples of visible images for both normal (a-b) and abnormal mode (c-h). It is certainly that the fiber splicer machine control unit treats visible images in order to align fibers and detect splicing abnormality using line detection technique; more specifically the Hough line detection.

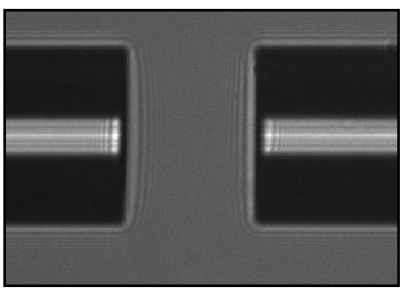

(a)

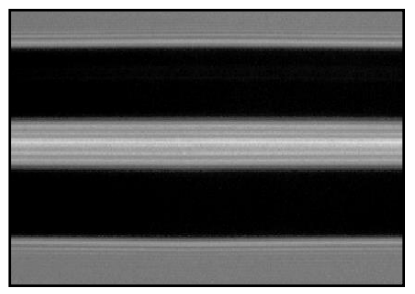

(b)

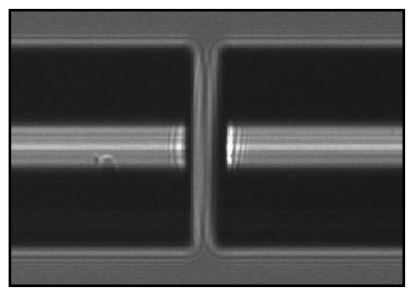

(c)

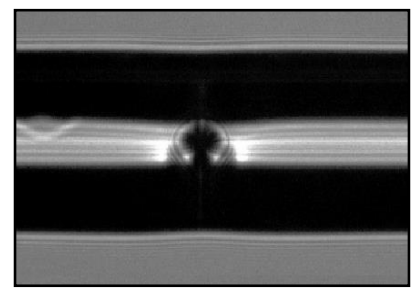

(d) 


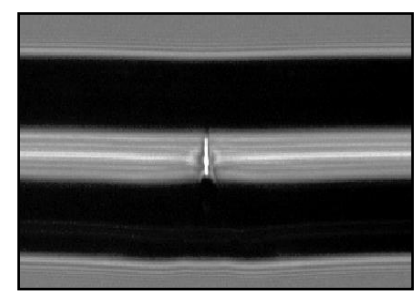

(e)

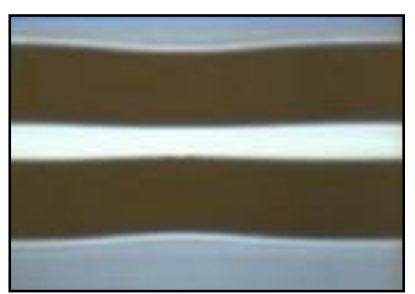

(f)

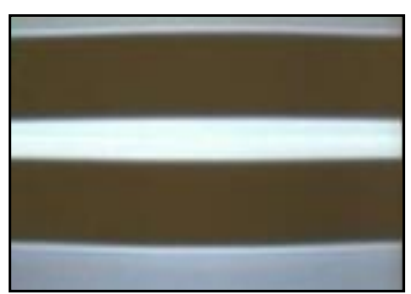

(g)

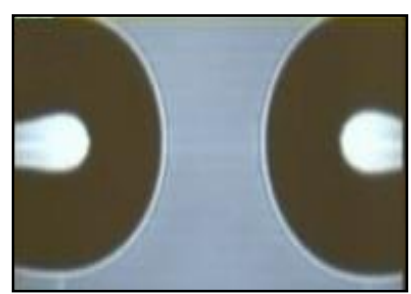

(h)

Fig. 3.Splicing samples of the visible fiber image: normal mode; a) in the beginning, b) at the end. Defect mode; c) large cleave angle, d) bubble, e) line, f) thin fiber, g) fat fiber,

h) separated fibers.

Fig.4 shows two image samples of normal mode (in the beginning and at the end of splicing process) and the line detection demands of the visible image. Six lines can be detected from the visible image of beginning; four horizontal with the aim of aligning fibers and detecting cores mismatch defect, and two vertical to compute cleave angles. For the visible image at the end of the splicing process, four horizontal lines are responsible to detect other defects described above. Hence, for the image in abnormal mode, the number of line detection result may be large than six (in the beginning) or four (at the end).

Moreover, the fiber splicer machine can estimate the splice loss which is a power metric of the input and the output light across the fiber. As the graph in Fig. 5 shows, the vast majority of splices were below $0.05 \mathrm{~dB}$, but there were several above that, as well as a few above $0.10 \mathrm{~dB}$. It should also be noted that splice studies performed in a lab, like those referenced in the graph, are usually done in ideal and relaxed conditions with state-of-the-art, well-maintained splicing equipment and cleavers. In reality, splicing is often done in haste, and in less than ideal conditions (cold, windy, dusty/dirty, etc.) with equipment that may be well used and not perform at its very best. Losses even greater than those seen in the splice studies here can be expected in field conditions.

Besides that, as shown in Fig. 6, a sample visible image with a good splice loss estimated by the fiber splicing machine but its corresponding hot (infra red) image looks poor by visual inspection. This refers that Denoising technique employed as preprocessing step before line detection may removes possibly important edge information of defect region [11]. By considering similar cases, we propose to use the data processing terminal and the splicing machine software to capture hot (infra red) images those will be processed for the purposes of splicing defect segmentation.
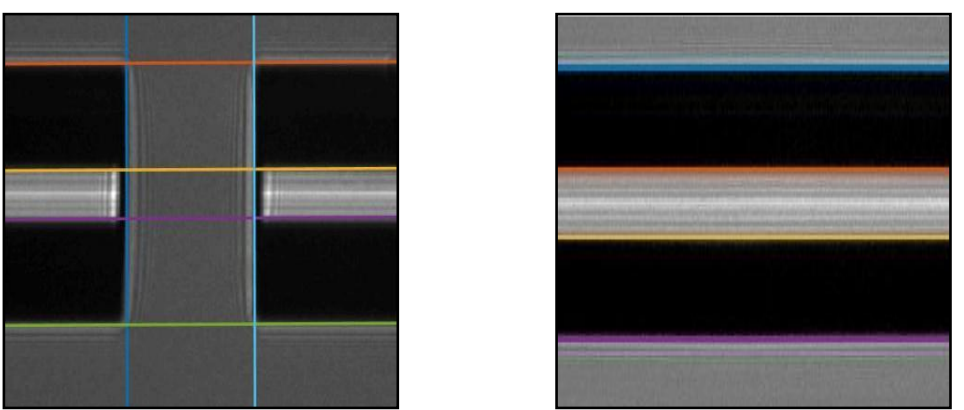

Fig. 4. Demonstration of line detection demands on normal mode samples. 


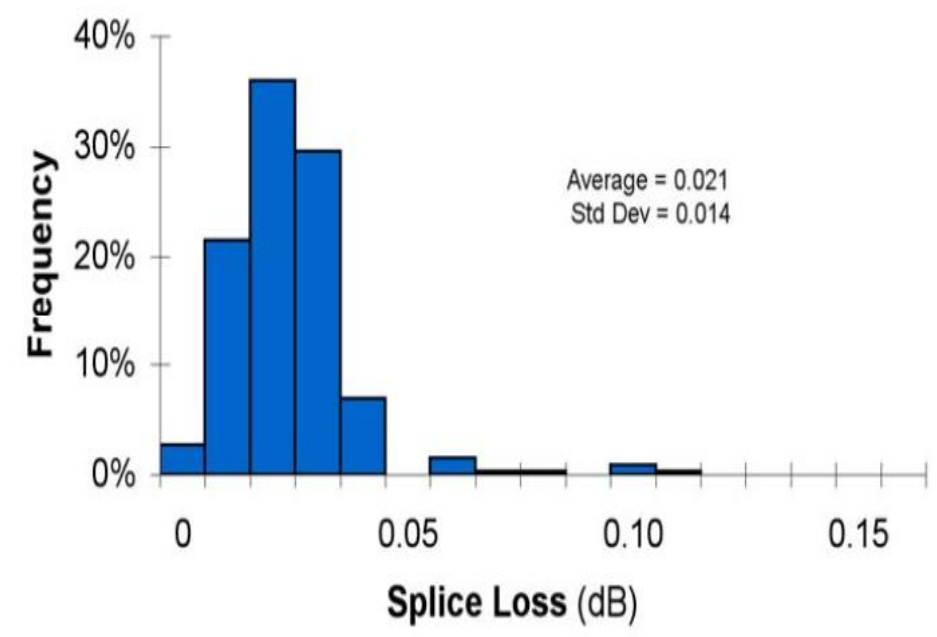

Fig. 5. Estimated Splice loss histogram.
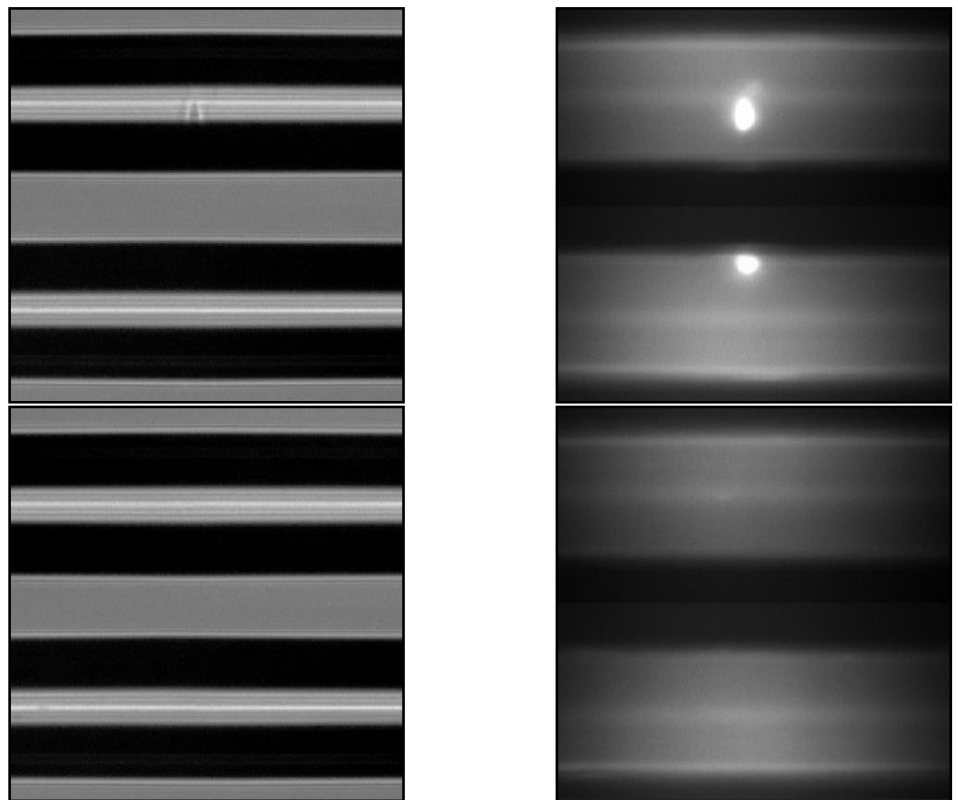

Fig. 6. The visible image samples and its corresponding hot image of the spliced fiber: (first row: defect sample. Second row: normal sample).

\section{Related works}

\subsection{CNN based models}

CNNs are among the most successful and widely used architectures in computer vision tasks, especially for image segmentation. A typical CNN, illustrated in Fig. 7, has a hierarchical structure and is composed of three type of layers to learn representations of data with multiple levels of abstraction [27]: i) convolutional layers, where a kernel (or filter) of weights is convolved in order to extract features; ii) nonlinear layers, which apply an activation function on feature maps (usually element-wise) in order to enable the modeling of non-linear functions by the network; and iii) pooling layers, which replace a small neighborhood of a feature map with some statistical information (mean, max, etc.) about the neighborhood and reduce spatial resolution [23]. Some of the most well-known CNN architectures include: UNet [20] and HRNet [22]. 


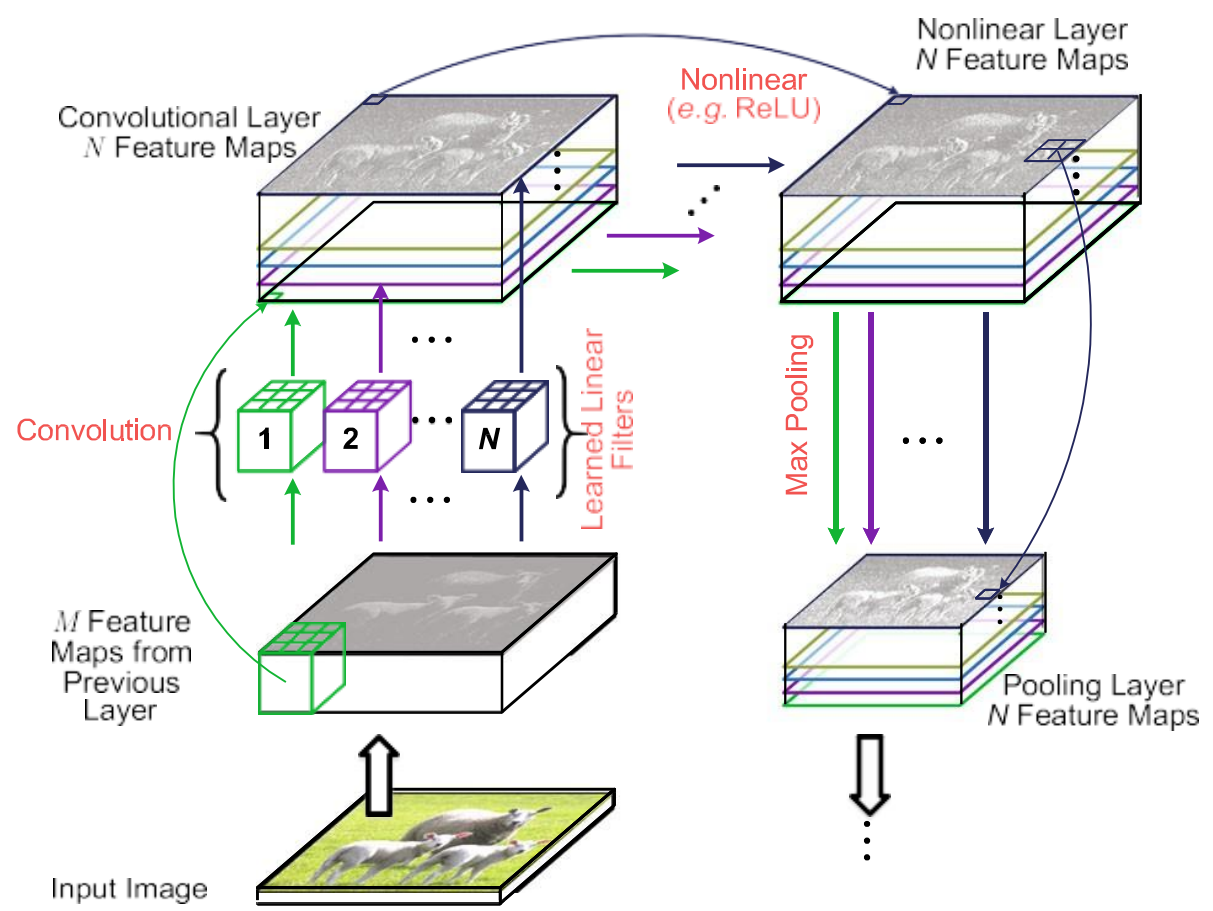

Fig. 7. Illustration of three operations that are repeatedly applied by a typical CNN: convolution with a number of linear filters; Nonlinearities (e.g. ReLU); and local pooling (e.g. max pooling) [27].

The UNet [20] architecture is proposed by taking the idea of the fully convolutional neural network (fCNN) [28]. The fCNN includes only convolutional layers, which enables it to take an image of arbitrary size and produce a segmentation map of the same size. The CNN architecture is modified by replacing all fully-connected layers with the fully-convolutional layers. As a result, the model outputs a spatial segmentation map instead of classification scores [23].

Authors in [20] took the idea of the fCNN one step further and proposed the UNet architecture, comprising a 'regular' fCNN followed by an up-sampling part where 'up'-convolutions are used to increase the image size, coined contractive and expansive paths [29].

Another popular model is the recently developed segmentation network, high-resolution network (HRNet) [22] as shown in Fig. 8.

Other than recovering high resolution representations as done in U-Net, HRNet maintains high-resolution representations through the encoding process by connecting the high-to-low resolution convolution streams in parallel, and repeatedly exchanging the information across resolutions [23].

The majority of CNNs based image segmentation research has focused on 2D Datasets which can be used for evaluating model performance.

\subsection{The GMM_GCM model}

Since the process of fiber splicing is accomplished in a dark environment, the LED lamp will cause a highlight region in the center of the fiber; so the image quality of the hot image (i.e. the contrast) is always low. Thus, in order to improve the image contrast, Liu et al [2,24] proposed to use the Gaussian Mixture Model GMM to estimate the illumination distribution. GMM is used to express the histogram as a sum of Gaussians using the Expectation Maximization (EM) algorithm. The GMM modeling of the histogram results in a number of Gaussians with each Gaussian being characterized by its mean, standard deviation and weight. The GMM model can be written as follow [30]:

$$
p\left(x \mid w_{i}, \mu_{i}, \sigma_{i}\right)=\sum_{i=1}^{k} w_{i} N_{i}\left(\mu_{i}, \sigma_{i}\right)
$$


where $k$ is the number of Gaussians, $w_{i}$ is the weight assigned to the $i^{\text {th }}$ Gaussian, $N_{i}$ represents the normalized $i^{\text {th }}$ Gaussian, and $\mu_{\mathrm{i}}, \sigma_{i}$ represents the mean and standard deviation of $i^{\text {th }}$ Gaussian.

When computing the GMM in this approach, firstly, its parameters are estimated by some selected normal mode image whose image qualities are good. Then the original image histogram is transferred into the log space by (2). The EM algorithm is used to estimate the GMM components of the image data in log space. Then, a kind of histogram transfer is given by (3).

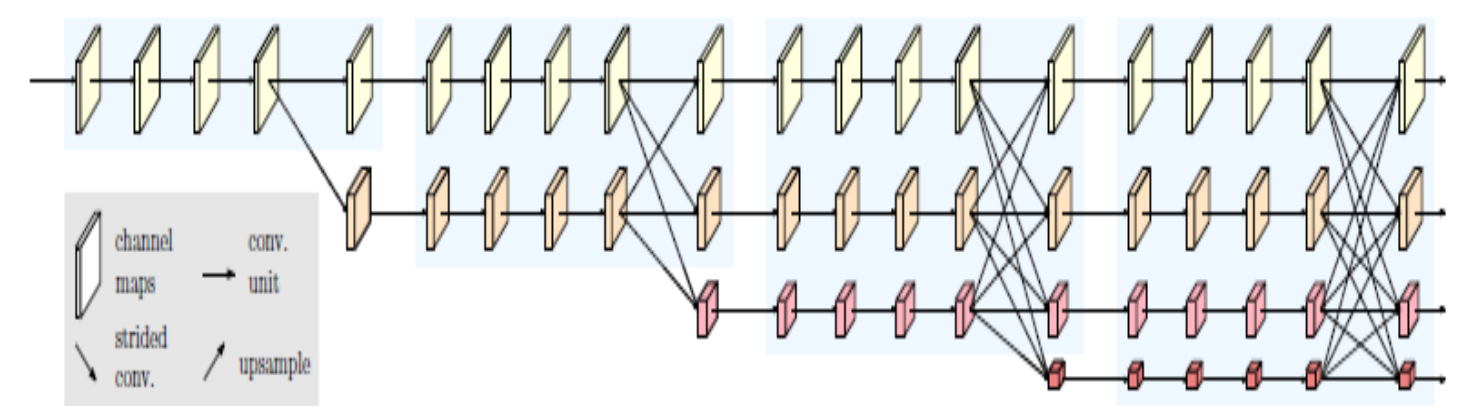

Fig. 8. Illustrating the HRNet architecture. It consists of parallel high-to-low resolution convolution streams with repeated information exchange across multi-resolution steams. There are four stages. The 1st stage consists of high-resolution convolutions. The 2nd (3rd, 4th) stage repeats two-resolution (three-resolution, four-resolution) blocks [22].

$$
\begin{gathered}
h^{\prime}=255 \times \frac{\log (h)-Y_{\min }}{Y_{\max }-Y_{\min }} \\
\left\{\begin{array}{l}
g\left(I^{\prime}\right)=I-t \exp (-\beta t) \\
t=\sum_{i=1}^{k} P_{i}\left(I^{\prime}\right) \mu_{i} \\
P\left(I^{\prime}\right)=\sum_{i=1}^{k} w_{i} N\left(I \mid \mu_{i}^{\prime}, \sigma_{i}\right) \\
\mu_{i}{ }^{\prime}=\exp \left(-\alpha_{i} \mu_{i}\right) \mu_{i}
\end{array}\right.
\end{gathered}
$$

where $I$ and $I^{\prime}$ are the image intensities in the log space. $Y_{\max }$ and $Y_{\min }$ represent the maximum and minimum luminance values of the log space of the image. $\mu_{i}{ }^{\prime}$ and $\mu_{i}$ are the means of GMM of the original image and that of the processed image respectively. $\sigma_{i}$ is the variance of the GMM of the original image. $h^{\prime}$ and $h$ are the histogram values of the original and computed images. $\alpha_{i}$ is a control constant which decides the degree of contrast adjustment. $\beta$ is also a control parameter of the histogram transfer [24].

After enhancing the contrast of hot image, the Graph Cut Model GCM [31] is used to segment defect region. The classic design method given by (4) employs the data dependent item (E1) and the smoothness item (E2). The data dependent item describes the cost of similarity between source (foreground) and sinking (background) vertexes; while the smoothness item calculates the cost of non-continuity among neighboring image pixels.

$$
E(A)=\lambda \sum_{i \in I} E 1\left(x_{i}\right)+(1-\lambda) \quad \sum_{(i, j) \in N, x_{i} \neq x_{j}} E 2\left(x_{i}, x_{j}\right)
$$

where the $E 1$ can be defined by Table I and (5). In Table I, symbol $C$ is a constant which is set by experiences. The $E 2$ can be estimated by (6). 
Table 1: Design of the E1 energy function of GCM

\begin{tabular}{lll}
\hline Type of Edge & Weight & Vertex Value \\
\hline \multirow{4}{*}{$p, s$} & $f_{i}^{F}$ & $p \notin F \cup B$ \\
& 0 & $p \in F$ \\
& $C$ & $p \in B$ \\
$\{p, t\}$ & $f_{i}^{B}$ & $p \notin F \cup B$ \\
& $C$ & $p \in F$ \\
& 0 & $p \in B$ \\
\hline
\end{tabular}

$$
\begin{gathered}
\left\{\begin{array}{l}
f_{i}^{F}=-\log \left[P\left(I_{i} \mid x_{i}=1\right)\right] \\
f_{i}^{B}=-\log \left[P\left(I_{i} \mid x_{i}=0\right)\right]
\end{array}\right. \\
E 2=\exp \left[-\frac{\left(I_{p}-I_{q}\right)^{2}}{2 \sigma_{n}^{2}}\right] \times \frac{1}{\operatorname{dist}(p, q)}
\end{gathered}
$$

where $I_{i}$ is the gray of pixel. Symbol $x_{i}$ equals 1 or o, which means the pixel belongs to foreground or background. Symbols $\mu$ and $\sigma$ are the means and the variances. Subscripts " $F$ " and " $B$ " represent the foreground and the background. Symbol $\sigma_{n}$ is the image noise. Symbol $\operatorname{dist}(p, q)$ is a distance metric.

Although GCM is one of the best choices for solving the image segmentation problem because of its good performance and high speed, however since the standard GMM parameters are just estimated by some selected normal mode image whose image qualities are good, and the contrast enhancement is related to some parameters (we refer here to control parameters $\alpha_{i}$ and $\beta$ in (3)); this makes not all the segmented region by GCM can be regarded as serious defects which will influence the final splicing effect. In addition, this approach employs the results of the region based flood fill method [32] segmentation as an initial segmentation of the GCM, and this may affect the final result. Moreover, the GCM cannot outperform other methods distinctly such as the active contour for some complex segmentation problems just because of its limit constraint ability (refers to $E 1$ and $E 2$ functions in (4)) [2].

\subsection{The RSF_LoG active contour}

It had been known that region-based Active Contours using local image statistics can deal effectively with highlight or intensity inhomogeneity problem, but they are found to act locally and to be easy to trap into local minima. To overcome these problems, the authors proposed in [18] a model that combines an optimized Laplacian of Gaussian (LoG) term which can smooth the homogeneous regions and enhance edge information and the Region-Scalable Fitting (RSF) term proposed in [13] which make use of local region information to drive the curve towards the boundaries. The total energy function can be defined as:

$$
E^{R S F S L}=E^{R S F}\left(\phi, f_{1}, f_{2}\right)+E^{O L}(\phi)
$$

where $\Phi$ is the level set function, $f_{1}, f_{2}$ are the interior, exterior local means, respectively giving in (9) and $E^{R S F}\left(\Phi, f_{1}, f_{2}\right)$ is the RSF energy defined in [13] as: 


$$
\begin{gathered}
E^{R S F}\left(\phi, f_{1}, f_{2}\right)=\lambda_{1} \int\left[\int_{\Omega} K_{\sigma}(x-y)\left|I(y)-f_{1}(x)\right|^{2} H_{\mathcal{E}}(\phi) d y+\right] \\
d x+\lambda_{2} \int\left[\int_{\Omega} K_{\sigma}(x-y)\left|I(y)-f_{2}(x)\right|^{2}\left(1-H_{\mathcal{E}}(\phi)\right) d y\right] d x, \\
f_{1}(x)=\frac{\int_{\Omega} K_{\sigma} *[I(x) H(\phi)]}{\int_{\Omega} K_{\sigma} * H(\phi)} \text { and } f_{2}(x)=\frac{\int_{\Omega} K_{\sigma} *[I(x)(1-H(\phi))]}{\int_{\Omega} K_{\sigma} *(1-H(\phi))}
\end{gathered}
$$

$E^{O L}(\Phi)$ is the optimized LoG energy defined as follow:

$$
\begin{gathered}
E^{O L}(\phi)=\omega L_{\Delta}(\phi)+v L(\phi)+\mu P(\phi)= \\
\omega \iint_{\Omega} H(\phi) L(x, y) d x d y \\
+v \iint_{\Omega} \delta(\phi)|\nabla(\phi)| d x d y \\
+\mu \iint_{\Omega}(|\nabla(\phi)|-1)^{2} d x d y
\end{gathered}
$$

The parameters $\omega, v$ and $\mu$ are the weight coefficients of each term. $L$ and $P$ are regularizers [10], $H(\Phi)$ and $\delta(\Phi)$ are the Heaviside and the Dirac function, respectively The $L_{\Delta}(\Phi)$ is optimized LoG term, where $L(x, y)$ is obtained by solving:

$$
\begin{aligned}
E^{L o G}(L)= & \iint_{\Omega} g(|\nabla I|)(L-0)^{2}+(1-g(|\nabla I|)) \\
& \left(L-\beta \Delta\left(G_{\sigma} * I\right)\right)^{2} d_{x} d_{y}
\end{aligned}
$$

where $L$ represents the value of optimized LoG of image, and $g(\nabla)=e^{-\alpha \nabla G \sigma^{*} I}, \alpha, \beta$ are positive constants. $g(\nabla I)$ is an edge indictor function. The values of $g(\nabla I)$ are small and approximately equal to $\mathrm{o}$ at the locations near the object boundaries, whereas, these values large and approximately equal to 1 in the homogeneous regions. $(L-O)^{2}$ is the data fitting term that measures the proximity between the optimized LoG and zero plane. When energy is descending, the term $g(\nabla I) \times(L-O)^{2}$ will drive $L$ close to $O$ in the homogeneous regions. Thus, it is helpful to smooth the homogeneous regions. Similarly, $\left(L-\beta \times \Delta\left(G_{\sigma}{ }^{*} I\right)\right)^{2}$ is the data fitting term that measures the proximity between the optimized LoG and the original LoG of image. The term $(1-g(\nabla)) \times\left(L-\beta \times \Delta\left(G_{\sigma}{ }^{*} I\right)\right)^{2}$ will drive $L$ close to $\Delta\left(G_{\sigma}^{*} I\right)$ at the locations near the object boundaries. Thus, it can preserve the object edges when $\beta$ is equal to 1 . And when $\beta$ is larger than 1, it can enhance the object edges [18]. By minimizing the energy in (11), the following Euler-Lagrange equation can be obtained:

$$
\left.\frac{\partial L}{\partial t}=g(|\nabla I|)\right) L-(1-g(|\nabla I|))\left(L-\beta \Delta\left(G_{\sigma} * I\right)\right)
$$

Using the steepest descent method to minimize the above energy functional in ( 7 ), the following gradient flow equation can be obtained:

$$
\begin{aligned}
\frac{\partial \phi}{\partial t}= & -\delta_{\varepsilon}(\phi)\left(\lambda_{1} e_{1}-\lambda_{2} e_{2}\right)+\omega \delta_{\varepsilon}(\phi) L \\
& +v \delta_{\varepsilon}(\phi) \operatorname{div}\left(\frac{\nabla_{\phi}}{\left|\nabla_{\phi}\right|}\right)+\mu\left(\Delta \phi-\operatorname{div}\left(\frac{\nabla_{\phi}}{\left|\nabla_{\phi}\right|}\right)\right)
\end{aligned}
$$


where $e_{1}(x)$ and $e_{2}(x)$ are defined as follow [18]:

$$
\begin{aligned}
& e_{1}(x)=\int_{\Omega} K_{\sigma}(x-y)\left|I(y)-f_{1}(x)\right|^{2} d_{y} \quad \text { and } \\
& e_{2}(x)=\int_{\Omega} K_{\sigma}(x-y)\left|I(y)-f_{2}(x)\right|^{2} d_{y}
\end{aligned}
$$

Combining edge and local region information improves the performance of active contour model, however the core element of edge information (i.e. the edge indicator function $g(\nabla I)$ ) has two major drawbacks: In practice, the discrete gradients are bounded and then, the function $g$ can be relatively far from zero on the edges and the curve may pass through the boundaries. The next issue is that for the noisy or textured regions, the image will have gradient maxima which induce local minima in the external energy. Therefore, the curve will not stop at the real object's boundaries. Alternatively, the Gaussian smoothing is used to remove spurious local minima. Yet, smoothing also removes possibly important edge information if the kernel width $\sigma$ is not chosen appropriately [11].

\section{The proposed model}

Our objective is to develop a model which is able to restrain highlight and to segment defects in hot images at the same stage with high accuracy. Different to the work proposed in [2,24] where the two previous stages (highlight restraint and segmentation) are separated, we have used a hybrid Active Contour based on the RSF-LoG model [18]. In addition, we substitute the edge indicator function based gradient information in (11) with a new one based on Local Binary Pattern LBP Proposed in [11,26] with the aim of overcoming edge leakage problem met with classical edge indicator based gradient information.

The LBP [33] operator has been applied in many active studies such as texture classification and face recognition [34]. The LBP operator combines characteristics of statistics and structural texture analysis; it describes the texture with primitives called textons [11].

The derivation of an LBP code is shown in Fig. 9a; taking a neighborhood of $3 \times 3$ of a central pixel, thresholding it into two levels "O" or " 1 " whether the neighbor of that pixel has smaller or larger value than the central pixel, respectively. An LBP code is obtained by multiplying the threshold values of eight pixels by binomial weights and summing up the result. Different texture primitives can be detected by the LBP code, Fig. 9b shows examples where ones and zeros are indicated with white and black circles respectively. A special kind of LBP, which will be used for edge detection, is called rotation-invariant uniform LBP where the number of bitwise $0 / 1$ and 1/o transitions in an LBP is only two or less. Nine classes of the uniform LBP are shown in Fig. 9c.

The new edge indicator function base LBP is resulting from the Canny edge detection with modified steps as follow [11]:

In the first step, unlike in Canny's algorithm where noise is suppressed by smoothing with Gaussian kernel, in this approach, a filter is generated which rejects pixel positions of LBPs which are likely to be produced by noise and accept the rest of classes; this classification is based on the work proposed in [26], where for some example images with different levels and types of artificial noise, LBP codes have been calculated and accumulated in LBP histograms. Each histogram has ten bins: nine for the uniform classes, and one for all other LBPs. From Fig. 10, it can be seen that the number of edges of different orientations (classes 2 to 6) decreases when the noise level increases while other LBPs (classes 0, 1, 7, 8 and 9) are much affected by noise. 


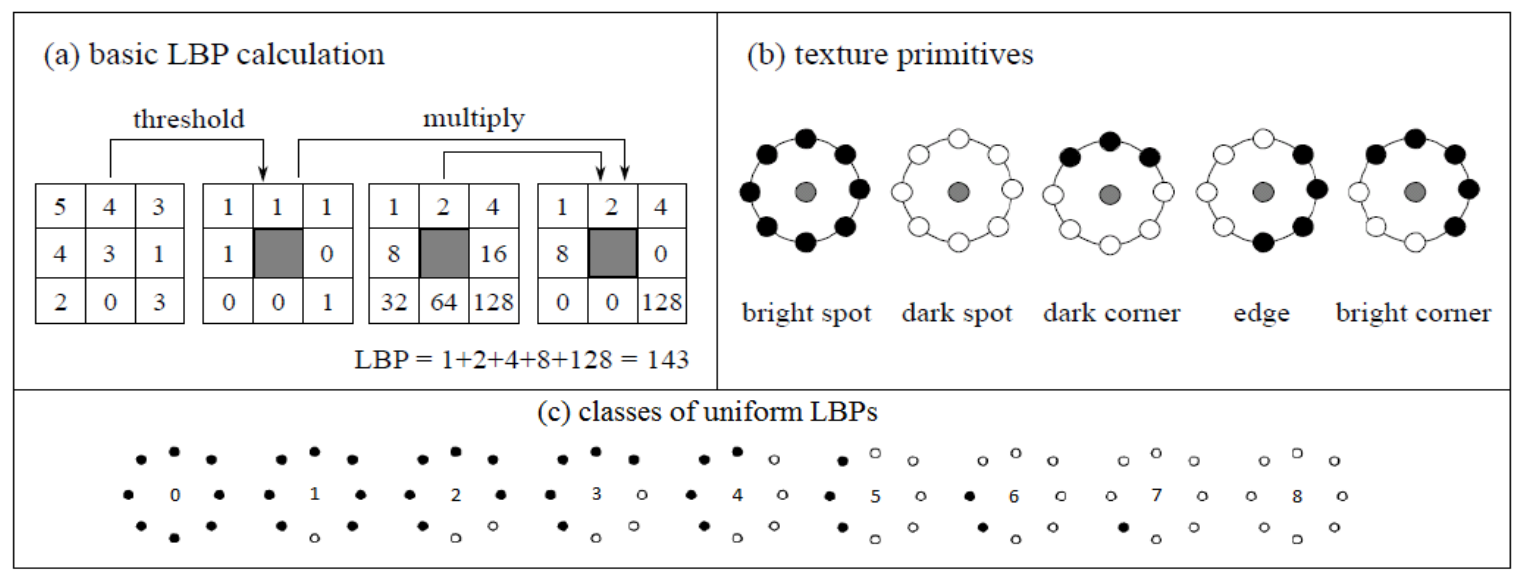

Fig. 9.Calculation, interpretation and uniform classes of LBPs.

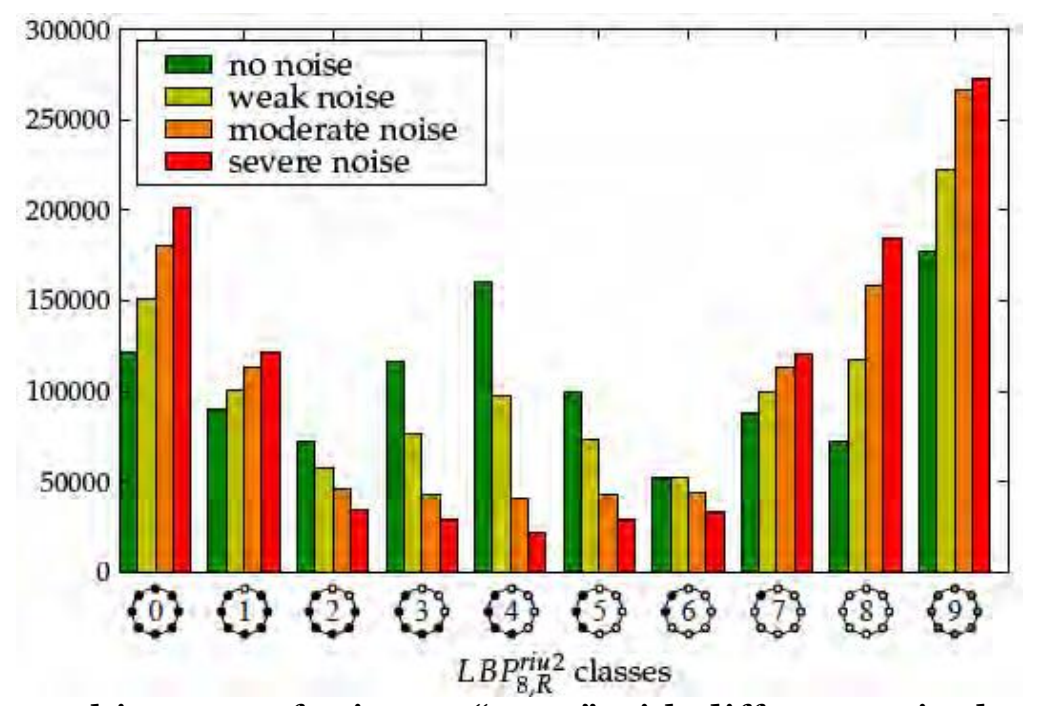

Fig. 10. LBP histogram for image "Lena" with different noise levels [26].

In the second step, gradient magnitudes in this approach are calculated at the accepted pixel positions using the local variance to increase the robustness against noise:

$$
\left\{\begin{array}{l}
G m=\sum_{r=1}^{3} \sqrt{V^{2} R_{P, r}} \\
V A R_{P, R}=\frac{1}{P} \sum_{p=0}^{P-1}\left(g_{p}-\mu\right)^{2} \\
\mu=\frac{1}{P} \sum_{p=0}^{P-1} g_{p} .
\end{array}\right.
$$

where $r$ is the variation of the radius $R$ to calculate several LBPs and summed up for the gradient magnitude in order to increase the robustness against noise, $P$ is the number of neighbours and $g_{p}$ are the gray values of surrounding pixels. Variance tends to focus too much on bright objects. So, standard-deviation is used instead of variance as it produces more homogeneous edge images.

In the third step, as in Canny's algorithm, four discretized gradient orientations: $0^{\circ}, 45^{\circ}, 90^{\circ}$, and $135^{\circ}$ are used. While these orientations are calculated using the atan2 function in Canny, this approach doesn't need to calculate anything in order to get the orientation. Instead, four sets: $\mathrm{D}^{0^{\circ}}{ }_{\mathrm{LBP}}, \mathrm{D} 45^{\circ}{ }_{\mathrm{LBP}}, \mathrm{D} 90^{\circ}{ }_{\mathrm{LBP}}$ and $\mathrm{D}^{135^{\circ}}{ }_{\mathrm{LBP}}$ of $\mathrm{LBP}$ that represent the orientations are simply defined. Each set D consists of $16 \mathrm{LBP}$ as shown in Fig. 11. 


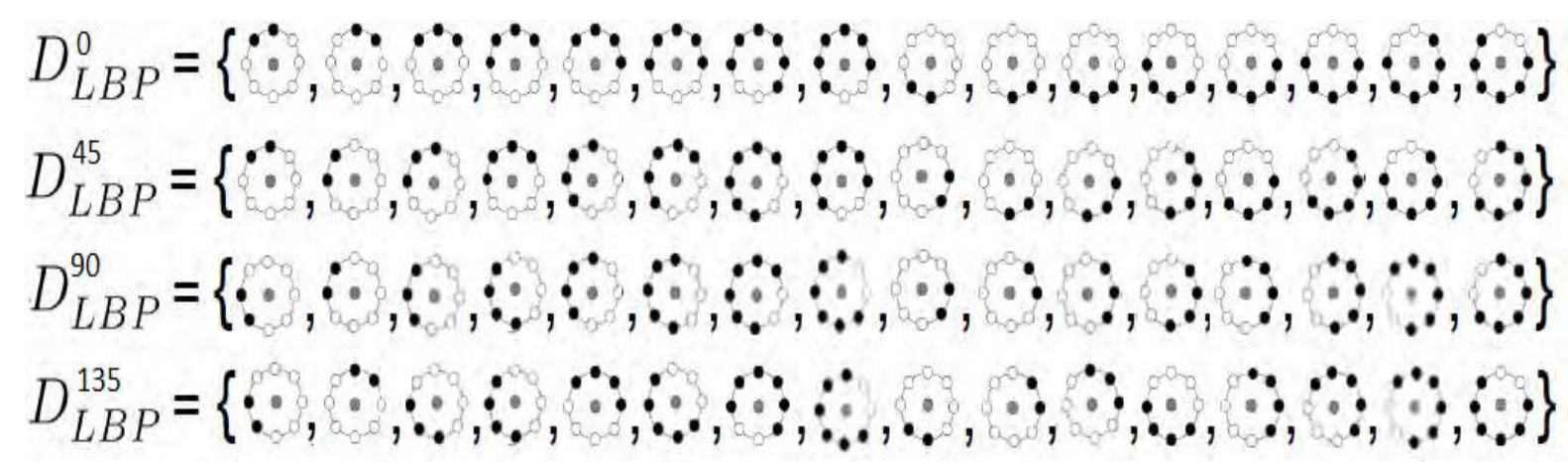

Fig. 11. The four sets with LBPs of different orientations [11].

The final step is to generate a binary edge pixel image $B$ using the hysteresis operator, in which pixels are marked as either edges, non edges and in-between, this is done based on two thresholds $t_{1}$ and $t_{2}$ with $t_{1}<t_{2}$. If a gradient magnitude exceeds $t_{2}$, it is accepted as edge pixel, while all pixels with gradient value less than $t_{1}$ are marked as non edges. The next step is to consider each of the pixels that are in-between, if they are connected to edge pixels these are marked as edge pixels as well. The result of this edge detector is the binary image $B$ in which the white pixels closely approximate the true edges of the original image.

The new edge indicator function based LBP is given by:

$$
g_{L B P}=1-B \text {, }
$$

The function $g_{L B P}$ presents the advantage that is made such as it is zero on edges whereas it is equal to one on flat and noisy regions and then, accordingly, the active contour will keep evolving in flat and noisy regions till it reaches the object boundaries [11].

Substituting the edge indicator $g$ in (12) with the new one based LBP $g_{L B P}$, the total energy function given in (13) becomes:

$$
\begin{aligned}
& \frac{\partial \phi}{\partial t}=-\delta_{\varepsilon}(\phi)\left(\lambda_{1} e_{1}-\lambda_{2} e_{2}\right)+\omega \delta_{\varepsilon}(\phi) L_{L B P} \\
& \quad+v \delta_{\varepsilon}(\phi) \operatorname{div}\left(\frac{\nabla_{\phi}}{\left|\nabla_{\phi}\right|}\right)+\mu\left(\Delta \phi-\operatorname{div}\left(\frac{\nabla_{\phi}}{\left|\nabla_{\phi}\right|}\right)\right)
\end{aligned}
$$

where ${ }^{L} L B P$ is the new optimized LoG based LBP.

\section{Experimental results}

In this section, in order to validate the performance of the proposed model, we apply and compare it with the original RSF-LoG model [18] and the GMM_GCM model [24] using three defect samples of hot images extracted from the splicer machine.

This study does not contain comparison with CNN based models including UNet [20] and HRNet [22] since such models require Image Dataset for evaluation.

All models are implemented using Matlab 9.5 in Windows 7; on 3.3 GHz Intel core i3 PC with 4GB of RAM.

Unless otherwise specified, we use the common parameters in the RSF_LoG [18] and the proposed model: $\sigma=1, \mu=2, v=0.006 \times 255^{2}, \omega=15, \lambda_{1}=\lambda_{2}=1$ and time step $\Delta t=0.1$. In the process of optimizing LoG, the parameters $\sigma=1, \alpha=0.01, \beta=5, \Delta t=0.01$ and the number of iterations is 250. For the edge detection method based LBP of the proposed model, the number of neighbors $P=8$, LBP radius $R=1$ and threshold parameters $t_{1}$ and $t_{2}$ are determined empirically according to images. For the GMM_GCM method [2], the component number of GMM $w=3$, the termination condition of the EM algorithm is set to 0.001 and the GCM result is getting from the Matlab Image Segmenter App.

Fig. 12 shows the comparison results where the first row shows the original hot images, images in second row are the ground truths of the defect segmentation validated by an expert, 
third row shows the results of the GMM_GCM model, images in fourth row are the results of original RSF-LoG model, while last row presents the results of the proposed model.
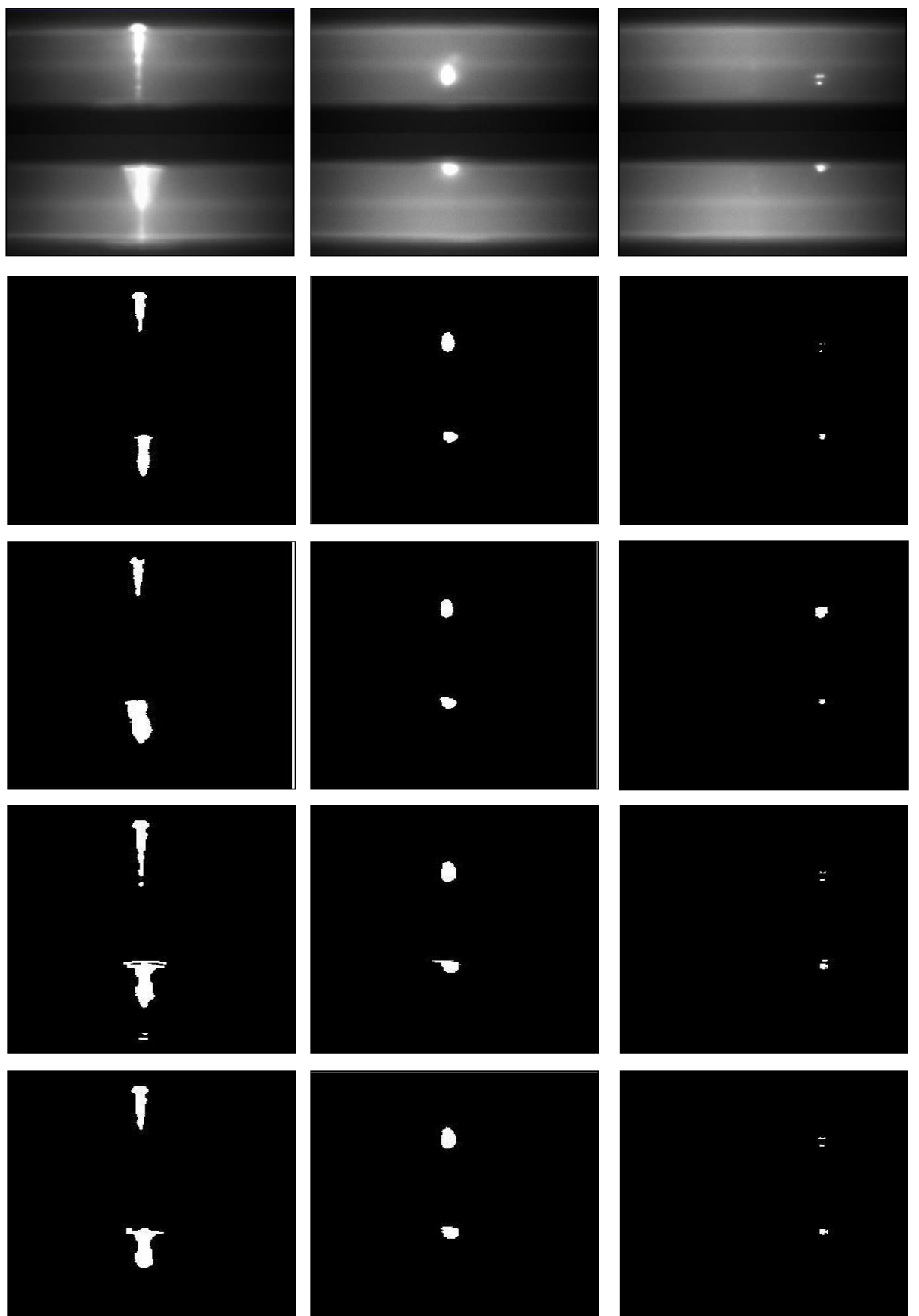

Fig. 12. Evaluation of segmentation results: first row: Original hot images. Second row: the ground truths of the defect segmentation. Third row: the results of the GMM_GCM model.

Fourth row: the results of the original RSF-LoG model. Last row: the results of the proposed model

The Dice coefficient [35] is used to compare and measure the segmentation accuracy. The Dice index $D \in[o, 1]$ between the obtained segmentation result $\mathrm{R}_{\mathrm{r}}$ and the ground truth $\mathrm{R}_{\mathrm{g}}$ is given by: $D\left(R_{r}, R_{g}\right)=\frac{2 \text { Area }\left(R r \cap R_{g}\right)}{\text { Area }\left(R_{r}\right)+\operatorname{Area}\left(R_{g}\right)}$. A higher Dice value (close to 1 ) indicates better segmentation performance. From the quantitative performance measure showed in Table 2, the proposed model shows excellent results with almost highest score of Dice index.

In particular, comparing the GMM_GCM model to the proposed model; refer to image of second column of Fig. 12 and its corresponding Dice value, the GMM works well in enhancing 
image contrast and restrain highlight (i.e. Intensity inhomogeneity). More specifically, Fig. 13 shows the original image histogram and the enhancing histogram after contrast enhancing using GMM. Since the defect region should be accentuated while the highlight region should be restrain, the GMM components in the right side can be weakened. However, the Estimation of GMM parameters play an important role and may affect segmentation accuracy by considering some intensities of background as parts of foreground or vice versa, and this is the case with the first and last column of Fig .12, where the proposed model outperforms the GMM_GCM model in term of segmentation accuracy.

Here the proposed model also outperforms the ACM_LoG due to the use of LBP edge detection method rather than the classical edge indicator function $g$; where no smoothing is necessary for the $g_{L B P}$, while the function $g(\nabla I)=e^{-\alpha \nabla G_{\sigma}{ }^{*} I}$ deals with the problem that smoothing with big filter size can suppress important edges and the curve will pass through them, but small filter size may not be sufficient to remove noise and then, the curve will stop evolving before reaching the real object boundaries. For more comparison, a visualized $g_{L B P}$ image of the sample image in the second column of Fig. 12 is contrasted with the classical $g$ image as shown in Fig. 14. Contrary to the edge indicator function $g$, it is clearly seen that the $g_{L B P}$ can accurately distinguish the object boundaries.

Table 2: Values of the Dice index (D) of the different models used in the study.

\begin{tabular}{llll}
\hline Image/ Models & $A C M L O G$ & $A C M \_L O G L B P$ & GMM_GCM \\
Image of first column & 0.7318 & $\mathbf{0 . 8 0 6 7}$ & 0.8040 \\
Image of second column & 0.8632 & 0.8770 & $\mathbf{0 . 9 0}$ \\
Image of third column & 0.6648 & $\mathbf{0 . 7 0 7 6}$ & 0.4162 \\
\hline
\end{tabular}
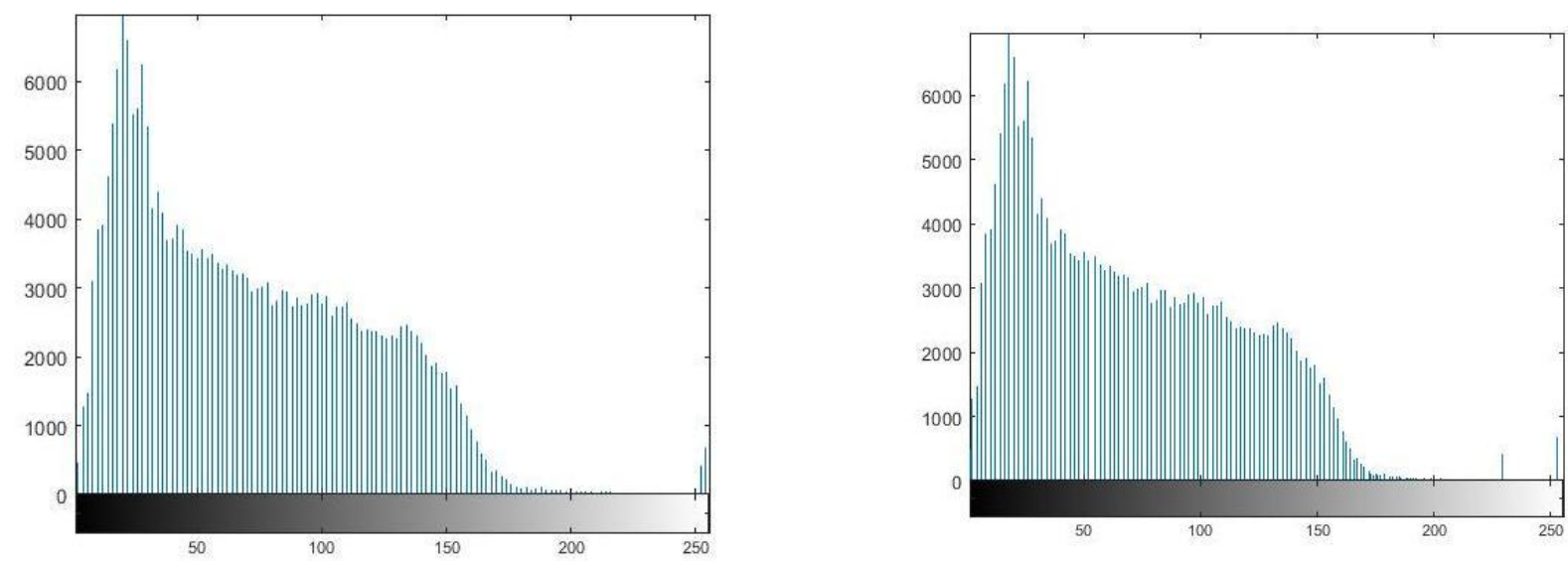

Fig. 13. Original histogram (left) and GMM histogram (right) of the image sample.

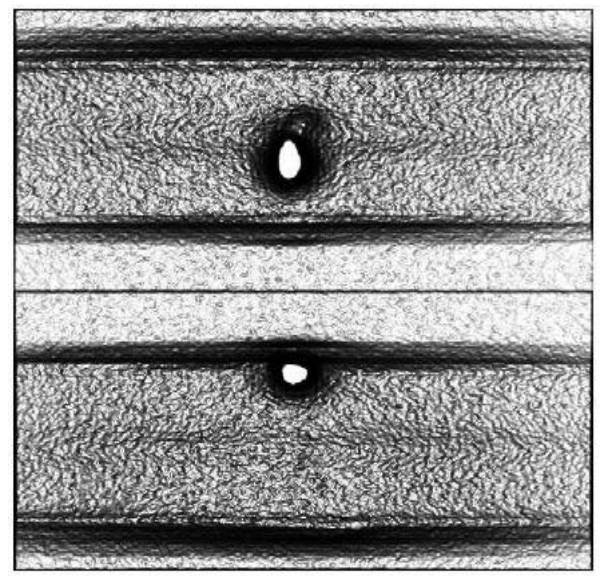

Fig. 14. The visualized classical edge function $g$ (left) and LBP edge function $g_{L B P}$ (right) of a sample image. 


\section{Conclusions}

In this paper, we propose a fiber splicing defect segmentation method for fiber splicer machine. We have used a hybrid edge and region active contour to achieve the highlight restraint and segmentation of defects at the same stage. In addition, a new edge indicator function based LBP is introduced with the aim of enhancing contour information. Experimental results demonstrate the potential of the proposed method in terms of segmentation accuracy. Future work will be devoted to build a feature dataset for splicing defect and extend the work based on CNN models.

\section{Acknowledgments}

The authors would like to thank all staff of CMSO (Centre Maintenance Support Optique) Algérie Telecom Biskra, for their help, their valuable comments concerning the fiber splicer machine. Authors would also like to thank Dr. ABDOLI Mohsen for helping us in implementing the GMM contrast enhancement method.

\section{References}

6. Meitzler, J.L., Rodriguez, M., Pradhan, S.K., Garren, J., Johnson, J., Watanabe, T., Mies, E. Is That Splice Really Good Enough? Improving Fiber Optic Splice Loss Measurement, 2003.

7. Liu, H., Wang, W., Gao, F., Li, J., Chen, K. Surface splicing defect analysis and application of polarization maintaining fiber using graph cut with illumination priors // Infrared Physics \& Technology, Vol. 66, 2014, pp. 125-135.

8. Mahmoudi, A., Regragui, F. Welding defect detection by segmentation of radiographic images // In 2009 WRI World Congress on Computer Science and Information Engineering, Vol. 7, 2009, pp. 111-115. IEEE.

9. Yazid, H., Arof, H., Yazid, H. Automated thresholding in radiographic image for welded joints // Nondestructive Testing and Evaluation, Vol. 27, № 1, 2012, pp. 69-80.

10. Anand, R. S., Kumar, P. Flaw detection in radiographic weld images using morphological approach // NDT \& E International, Vol. 39, № 1, 2006, pp. 29-33.

11. Boutiche, Y. Local Segmentation via an Implicit Region-Based Deformable Model Applied To Weld Defects Extraction // International Journal of Computer and Information Technology, Vol. 2, № 4, 2013, pp. 815-820.

12. Boutiche, Y., Halimi, M. Automatic Detection and Features Computation of Weld Defects for Radiographic Inspection // In International Conference on NDT and Materials Industry and Alloys (IC-WNDT-MI'14), 2014.

13. Boutiche, Y.. Fast Level Set Algorithm for Extraction and Evaluation of Weld Defects in Radiographic Images // In Artificial Intelligence and Computer Vision, 2017, pp. 51-68. Springer, Cham.

14. Caselles, V., Kimmel, R., Sapiro, G. Geodesic active contours // International journal of computer vision, Vol. 22, № 1, 1997, pp. 61-79.

15. Li, C., Xu, C., Gui, C., Fox, M. D. Distance regularized level set evolution and its application to image segmentation // IEEE transactions on image processing, Vol. 19, № 12, 2010, pp. 3243-3254.

16. Azizi, A., Elkourd, K., Azizi, Z. Robust Active Contour Model Guided by Local Binary Pattern Stopping Function // Cybernetics and Information Technologies, Vol. 17, № 4, 2017, pp. 165-182.

17. Chan, T. F., Vese, L. A. Active contours without edges // IEEE Transactions on image processing, Vol. 10, № 2, 2001, pp. 266-277.

18. Li, C., Kao, C. Y., Gore, J. C., Ding, Z. Minimization of region-scalable fitting energy for image segmentation // IEEE transactions on image processing, Vol. 17, № 10, 2008, pp. 1940-1949. 
19. Zhang, K., Zhang, L., Lam, K. M., Zhang, D. A level set approach to image segmentation with intensity inhomogeneity // IEEE transactions on cybernetics, Vol. 46, № 2, 2015, pp. 546-557.

20.Azizi, A., Elkourd, K. Fast Region-based Active Contour Model Driven by Local Signed Pressure Force // ELCVIA: electronic letters on computer vision and image analysis, Vol. 15, № 1, 2016, pp. 1-13.

21. $\mathrm{Xu}, \mathrm{H}$., Liu, T., Wang, G. Hybrid geodesic region-based active contours for image segmentation // Computers \& Electrical Engineering, Vol. 40, № 3, 2014, pp. 858-869.

22. Abdallah, A., Kaouther, E. A Hybrid Active Contour without Re-initialization // In Proceedings of the International Conference on Intelligent Information Processing, Security and Advanced Communication, p. 45, 2015, ACM.

23. Ding, K., Xiao, L., Weng, G. Active contours driven by region-scalable fitting and optimized Laplacian of Gaussian energy for image segmentation // Signal Processing, Vol. 134, 2017, pp. 224-233.

24. Azizi, A. Détection du Contour Actif de Différentes Images. // Doctoral dissertation, Université Mohamed Khider-Biskra, 2017.

25. Ronneberger, O., Fischer, P., Brox, T. U-net: Convolutional networks for biomedical image segmentation // In International Conference on Medical image computing and computer-assisted intervention, 2015, pp. 234-241. Springer, Cham.

26.Xiao, T., Liu, Y., Zhou, B., Jiang, Y., Sun, J. Unified perceptual parsing for scene understanding // In Proceedings of the European Conference on Computer Vision (ECCV), 2018, pp. 418-434.

27. Sun, K., Zhao, Y., Jiang, B., Cheng, T., Xiao, B., Liu, D., Wang, J. High-resolution representations for labeling pixels and regions // arXiv preprint, 2019, arXiv:1904.04514.

28. Minaee, S., Boykov, Y., Porikli, F., Plaza, A., Kehtarnavaz, N., Terzopoulos, D. Image segmentation using deep learning: A survey // arXiv preprint, 2020, arXiv:2001.05566.

29. Liu, H., Wang, W., Li, X., Li, F. Defect segmentation of fiber splicing on an industrial robot system using GMM and graph cut // In 2012 IEEE International Conference on Robotics and Biomimetics (ROBIO), 2012, pp. 1968-1972. IEEE.

30.Abdoli, M., Sarikhani, H., Ghanbari, M., Brault, P. Gaussian mixture model-based contrast enhancement // IET image processing, Vol. 9, № 7, 2015, pp. 569-577.

31. Teutsch, M., Beyerer, J. Noise resistant gradient calculation and edge detection using local binary patterns // In Asian Conference on Computer Vision, 2012, pp. 1-14, Springer, Berlin, Heidelberg.

32. Liu, L., Ouyang, W., Wang, X., Fieguth, P., Chen, J., Liu, X., Pietikäinen, M. Deep learning for generic object detection: A survey // International journal of computer vision, Vol. 128 № 2, 2020, pp. 261-318.

33. Long, J., Shelhamer, E., Darrell, T. Fully convolutional networks for semantic segmentation // In Proceedings of the IEEE conference on computer vision and pattern recognition, 2015, pp. 3431-3440.

34. Litjens, G., Kooi, T., Bejnordi, B. E., Setio, A. A. A., Ciompi, F., Ghafoorian, M., Sánchez, C. I. A survey on deep learning in medical image analysis // Medical image analysis, Vol. 42, 2017, pp. 60-88.

35. Mohanty, K. K., Gellaboina, M. K. Enhancement of low light image based on Gaussian mixture modeling // In 2010 2nd European Workshop on Visual Information Processing (EUVIP), 2010, pp. 232-236, IEEE.

36. Boykov, Y. Y., Jolly, M. P. Interactive graph cuts for optimal boundary \& region segmentation of objects in ND images // In Proceedings eighth IEEE international conference on computer vision (ICCV 2001), 2001, Vol. 1, pp. 105-112. IEEE.

37. Fathi, M., Hiltner, J. A new fuzzy based flood-fill algorithm for 3D NMR brain segmentation // In IEEE SMC'99 Conference Proceedings. 1999 IEEE International Conference on Systems, Man, and Cybernetics (Cat. No. 99CH37028), Vol. 4, 1999, pp. 881-885, IEEE. 
38.Ojala, T., Pietikäinen, M., Mäenpää, T. Multiresolution gray-scale and rotation invariant texture classification with local binary patterns // IEEE Transactions on Pattern Analysis \& Machine Intelligence, Vol. 7, 2002, pp. 971-987.

39.Ammar, C., Mebarka, B., Abdelmalik, O., Salah, B. Evaluation of Histograms Local Features and Dimensionality Reduction for 3D Face Verification // JIPS (Journal of Information Processing Systems), Vol. 12, № 3, 2016, pp. 468-488.

40.Mukherjee, S., Acton, S. T. Region based segmentation in presence of intensity inhomogeneity using legendre polynomials // IEEE Signal Processing Letters, Vol. 22, № 3, 2014, pp. 298-302. 